\title{
Dismantling institutional racism: theory and action
}

\author{
Derek M. Griffith - Mondi Mason - Michael Yonas • \\ Eugenia Eng · Vanessa Jeffries · Suzanne Plihcik · \\ Barton Parks
}

Published online: 3 April 2007

(C) Springer Science+Business Media, LLC 2007

\begin{abstract}
Despite a strong commitment to promoting social change and liberation, there are few community psychology models for creating systems change to address oppression. Given how embedded racism is in institutions such as healthcare, a significant shift in the system's policies, practices, and procedures is required to address institutional racism and create organizational and institutional change. This paper describes a systemic intervention to address racial inequities in healthcare quality called dismantling racism. The dismantling racism approach assumes healthcare disparities are the result of the intersection of a complex system (healthcare) and a complex problem (racism). Thus, dismantling racism is a systemic and systematic intervention designed to illuminate where and how to intervene in a given healthcare system to address proximal and distal factors associated with healthcare
\end{abstract}

D. M. Griffith $(\bowtie)$

Department of Health Behavior \& Health Education,

School of Public Health, University of Michigan, 1420

Washington Heights Drive SPH II, Ann Arbor,

MI 481092029, USA

e-mail: derekmg@umich.edu

\section{Mason}

Association for the Study and Development of Community, Gaithersburg, MD, USA

M. Yonas · E. Eng

University of North Carolina, Chapel Hill, NC, USA

V. Jeffries

Rural County Public Health Department, Pittsboro, NC, USA

\section{S. Plihcik}

Dismantling Racism Works, Smyrna, GA, USA

S. Plihcik · B. Parks

The Partnership Project, Greensboro, NC, USA disparities. This paper describes the theory behind dismantling racism, the elements of the intervention strategy, and the strengths and limitations of this systems change approach.

Keywords Racism - Systems change $\cdot$ Soft systems methodology · Healthcare disparities - Health disparities . Institutional racism - Health care - Health inequities . Healthcare inequities

\section{Introduction}

While the Civil Rights Era eradicated the more overt racial and ethnic barriers in the US health care system, over 40 years later the nation is still struggling with how best to address inequities that violate our most fundamental professional and national principles (Smith, 1999). According to Dr. Martin Luther King, Jr., "Of all forms of inequity, injustice in healthcare is the most shocking and inhumane" (King, 1966, March 25). Racial and ethnic differences in healthcare quality are so surprising and troubling because they violate physicians' and health care workers' conscious commitment to equity and helping those in need (Geiger, 2006). Despite reviews of hundreds of studies across diseases illustrating the breadth and depth of healthcare disparities (Geiger, 2006; Smedley, Stith, \& Nelson, 2003; Sohler, Walmsley, Lubetkin, \& Geiger, 2003), professionals have been reluctant to believe that their own behaviors, those of their peers, and the policies of their institutions may often go against their professional oaths and principles (Geiger, 2006; Smedley et al., 2003).

Racial and ethnic healthcare disparities are defined as differences in the quality of healthcare that is provided to 
patients of color when compared with white patients (Smedley et al., 2003). These differences are not accounted for by access-related factors, clinical needs, insurance status, treatment refusal rates or the appropriateness of the intervention, and occur across preventative, ameliorative and supportive services. The question of why these disparities exist and how they can be addressed continues to perplex practitioners and policy makers. The most common assumption is that racial and ethnic disparities in healthcare are the result of providers' lack of cultural competence (Horner et al., 2004). Therefore, the most common approach to addressing racial and ethnic disparities in healthcare quality has been through individual-level reeducation (i.e., in-services, cultural competence training, and educational sessions to increase knowledge of different cultural groups). These efforts, however, have shown limited effectiveness in reducing healthcare disparities (King, 1996; Wyszewianski \& Green, 2000). In contrast to this approach, the Sullivan Commission (2004) suggests that an essential starting point for appreciating the complexity of disparities in today's health care system is to recognize the existence of race-based inequities in health care delivery, and then to identify how racism operates throughout the health care system.

This paper describes an emerging model for addressing healthcare disparities called dismantling racism. We begin by arguing that a systems change approach is necessary to reduce and eventually eliminate healthcare disparities by illustrating how healthcare disparities are rooted in institutional racism. We then synthesize concepts from community psychology, social work, sociology and public health to describe the theoretical framework that underlies the dismantling racism approach. The theoretical framework for dismantling racism is an anti-racist community organizing model that incorporates elements of power, sociopolitical development and empowerment theory. We conclude with a description of the strategies and processes that comprise the dismantling racism intervention and a discussion of the challenges and limitations of this approach as implemented in two settings: a county public health department and an urban medical system.

Why a systems change approach to address healthcare disparities?

Systems change approaches are recommended when organizations and institutions face complex problems that require systematic, multi-level change (Midgley, 2006). A common assumption of systems change approaches is that everything in the universe is directly or indirectly connected to everything else. However, because there are limits to what we know about any situation and problem, comprehensive analysis is impossible. Systems change approaches provide a foundation for defining boundaries and making value judgments to determine who and what will be included in an intervention. These boundaries are set so that a wide array of stakeholder values and concerns can be accounted for and considered without compromising comprehension of the problem.

The dismantling racism approach assumes healthcare disparities are the result of the intersection of a complex system (healthcare) and a complex problem (racism). A systems change approach is warranted to address healthcare disparities for three basic reasons: (1) the problem of healthcare disparities is rooted in a history of racism and segregation in medicine and healthcare; (2) healthcare is one of many societal institutions that provides services of unequal quality and resources to People of Color when compared with Whites; (3) the complexity of this history and system suggests that the level of intervention (healthcare institutions) must match the level of conceptualization of the problem (institutional racism). Each of these points will be discussed in more detail below.

The problem of healthcare disparities

Healthcare disparities are outcomes at the nexus of the patient-provider interaction. Patients of color are more likely than Whites to perceive bias and lack of cultural competence when seeking treatment in the health care system. These perceptions of discrimination diminish but persist even when controlling for demographic factors, health literacy, self-rated health status, source of care and reports of medical communication (Johnson, Saha, Arbelaez, Beach, \& Cooper, 2004). Other studies have found that perceived discrimination was associated with lower levels of satisfaction with the health care system (LaVeist, Nickerson, \& Bowie, 2000; Saha, Arbelaez, \& Cooper, 2003). Perceived discrimination also has been found to affect patients' utilization of health services, particularly for chronic diseases. Blanchard and Lurie (2004) found that People of Color who perceived disrespect or discrimination because of their race were less likely to get an annual physical exam, to receive appropriate preventive care for heart disease, hypertension or diabetes, and to follow a doctor's advice. Thus, patients' perceptions of discrimination can be a major barrier to the effective management of disease, particularly for diseases that require collaboration between the patient and provider (e.g., diabetes).

As was stated earlier, the most common approach to addressing healthcare disparities has been to promote cultural competency or some other form of training to increase cultural awareness and reduce racial insensitivity (Horner et al., 2004; King, 1996). These educational approaches have demonstrated limited effectiveness in reducing or eliminating healthcare disparities, especially when they are 
not coupled with policy or organizational change efforts (Green, Gorenflo, \& Wyszewianski, 2002; Wyszewianski \& Green, 2000). Another popular tact has been improving overall quality of care and adherence to evidence-based clinical guidelines (Baquet, Carter-Pokras, \& BengenSeltzer, 2004; Lavizzo-Mourey \& Jung, 2005; Taylor \& Lurie, 2004). Though this approach has helped to improve the overall quality of health care, universal approaches to improving overall quality and access can have the unintentional effect of widening disparities (Ceci \& Papierno, 2005). The two primary problems with these approaches are that they do not consider the nature, root or scope of healthcare disparities.

Historically, the US healthcare system has not provided equitable care to all of its clients (Smedley et al., 2003; Trubek \& Das, 2003). Research on the quality of healthcare provided for cardiovascular disease (Kressin \& Petersen, 2001; Lillie-Blanton, Maddox, Rushing, \& Mensah, 2004), cancer (Shavers \& Brown, 2002), pediatric diseases (Hahn, Ostermann, Richter, \& David, 1995; Stevens \& Shi, 2002), and across diseases (Smedley et al., 2003; Sohler et al., 2003) has consistently found evidence that People of Color receive lower quality health care (Geiger, 2006). These disparities have not just been found for high-technology interventions, but they also have been found for routine medical procedures, exams, and care across preventative, ameliorative and supportive services (Geiger, 2006; Smedley et al., 2003). Though a number of studies have found that black-white disparities in some medical services have narrowed over time (AHRQ, 2005; Trivedi, Zaslavsky, Schneider, \& Ayanian, 2005; Escarce \& McGuire 2004), the extent of healthcare disparities remains vast and rooted in the history of race and medicine.

Reviews of the history of race and medicine have concluded that racism is-at least in part-responsible for the fact that since arriving as slaves, African Americans have had the worst heath care, the worst health status, and the worst health outcomes of any racial or ethnic group in the US (Byrd \& Clayton, 2000; Krieger, 1987). Racism has been defined as "an organized system, rooted in an ideology of inferiority that categorizes, ranks, and differentially allocates societal resources to human population groups" (Williams \& Rucker, 2000, p. 76). This definition helps to explain why, for example, Black Americans continue to lag behind Whites on almost every measure of prosperity (i.e., employment, criminal justice, economic resources, health, and education) (Pettigrew, 2004). Racism is not to be confused with other forms of oppression. According to Aptheker (1992), "There are common ingredients in all... But belief in the superiority of one's particular culture, or nation or class or sex is not the same as belief in the inherent, immutable, and significant inferiority of an entire physically characterized people, particularly in mental capacity, but also in emotional and ethical features"'(pp.xiii-xiv as cited in Jones, 1997, p.515).

Conventional wisdom in the US has held that there were biologically and genetically distinct human races, and for centuries these notions have been reinforced by pseudoscientific articles in the medical literature (Freeman, 2003; Geiger, 2006). Presumed racial differences were based on visible traits (skin color, facial features, hair type, etc.) and suggested that African Americans, southern and eastern Europeans, Chinese, and darker-skinned immigrants of other nationalities were biologically and intellectually inferior and more susceptible to disease (Griffith, Moy, Reischl, \& Dayton, 2006). In addition, prior to the last four decades, the American healthcare system was segregated by race and class (Geiger, 2006). In healthcare, it was legal and often customary for hospitals to refuse treatment to African Americans or to house them separately in inferior, under-funded, and often overcrowded basement wards and other facilities. There was also considerable discrimination by medical and health professionals who furnished care and ultimately determined the structure, design, and operation of the health system (Geiger, 2006). The beliefs about the inherent inferiority of People of Color and the structures that were created to provide inferior treatment to them illustrate how racism became institutionalized in the science and practice of medicine including the US healthcare system.

\section{Theoretical foundations of dismantling racism}

The history of racism in medicine and healthcare described earlier illustrates that the problem of healthcare disparities is not simply one of individual behavior. It is a problem that is rooted in organizational and institutional structures and practices (Byrd \& Clayton, 2000, 2001). Given how embedded racism is in institutions such as healthcare, a significant shift in the system's policies, practices, and procedures is required to address institutional racism and create organizational and institutional change to reduce healthcare disparities. Arguably, it is impossible to fully conceptualize the breadth, depth and complexity of racism and its relationship to healthcare disparities, yet it is necessary to identify where and how to intervene. Dismantling racism, therefore, is a systemic and systematic intervention process designed to illuminate where and how to intervene in a given healthcare system to address healthcare disparities.

Systemic interventions are purposeful actions to create change in relation to and reflection upon boundaries (Midgley, 2006). In settings and for problems where comprehensive analysis is impossible, systems interventions provide a systematic process for considering the 
diversity of factors, perspectives, and issues that underlie a problem and that may be effective intervention strategies (Midgley, 2006). One of the primary advantages of systems interventions is its synergy of boundary critique and methodological and theoretical pluralism. Boundary critique is a dialogue that establishes the broad parameters of who and what will be included in an intervention, and includes all affected and interested stakeholders. Because this process is driven by the values and value judgments of interested parties, it is important that the largest set of stakeholders that can be accounted for without sacrificing effective communication or comprehension is included. Methodological and theoretical pluralism is the process of drawing upon and mixing methods and theories because no one theory or method is sufficiently comprehensive. The integration of boundary critique and methodological pluralism helps to correct the potential weaknesses of each individual theory or strategy (Midgley, 2006). The dismantling racism intervention, therefore, is based on the integration of three theoretical and methodological approaches: institutional racism, anti-racist community organizing, and Soft Systems Methodology. Each will be discussed briefly in turn.

\section{Institutional racism}

Institutional racism describes how institutional structures and processes organize and promote racial inequity (Jones, 1997). "These effects are suffused throughout the culture via institutional structures, ideological beliefs, and personal everyday actions of people in the culture, and these effects are passed on from generations to generations" (Jones, 1997, p. 472). Institutional racism represents "the collective failure of an organization to provide an appropriate and professional service to people because of their color, culture, or ethnic origin. It can be seen or detected in processes, attitudes and behaviors which amount to discrimination through unwitting prejudice, ignorance, thoughtlessness and racist stereotyping which disadvantage minority ethnic people"' (MacPherson, 1999, p. 28 as cited in Gillborn, 2005, p. 498). Griffith and colleagues (2007) argue that institutional racism can be conceptualized at three levels of an organization: the extraorganizational, the intraorganizational, and the individual. At the extraorganizational level, institutional racism explains the reciprocal relationship between organizations and their external environment. At the intraorganizational level, institutional racism operates through an organization's internal climate, policies and procedures. These include the relationships among staff, which are rooted in formal and informal hierarchies and power relationships. Finally, at the individual level, racism operates through staff members' attitudes, beliefs, and behaviors.

\section{Anti-racist community organizing}

Anti-racist community organizing is an intervention strategy that builds on the core components and principles of community organizing and infuses anti-racism as a core value and belief. Anti-racism is the advocacy of individual conduct, institutional practices, and cultural expressions that promote inclusiveness and interdependence and acknowledgement and respect racial differences (Jones, 1997). Anti-racist approaches to organizing assume that cultural and institutional structures have created an unequal system, and suggest that the solution is to change the institutions, organizations, and individuals within these contexts (Shapiro, 2002). The community organizing strategy for creating change is to reduce inequities in power relations and address the root causes of social problems (Wittig, 1996). Anti-racist organizing seeks to bring people together who are affected by the problem to increase their collective power so they can resolve the problem, and to hold those in power accountable to principles of justice and equity (Jones, 2003). Anti-racist organizing efforts bring people together to more effectively coordinate and work together, making them more powerful actors in their lives rather than passive objects of decisions made by others (Jones, 2003; Neighborhood Funders Group, 2001).

Community organizing consists of four interrelated phases: assessment, research, action, and reflection (Speer \& Hughey, 1995). Assessment is the process of identifying the issues affecting a system, usually conducted through one-on-one conversations with key members of a community or organization. In addition to gathering information, the conversations are opportunities to deepen relationships among community members. The research phase is the opportunity to identify the potential causes of the issues identified in the assessment phase. Anti-racist organizing approaches suggest that creating social and institutional change through community organizing must be rooted in a common, critical analysis of structural and institutional racism, which includes understanding different types, manifestations, and faces of power (Shapiro, 2002). The research phase also includes gathering information regarding the nature of the issue and its potential influences and solutions. The key to this phase is uncovering how power and racism are made manifest in this context, while gaining an understanding of the organizational infrastructure, mission and functions (Griffith et al., 2007). Without understanding both the metrics of institutional racism and the basic goals and objectives of the organization, the intervention is likely to fail. The action phase is an effort to exercise power developed through organizing. The action process incorporates strategizing and mobilizing resources for collective action. It is critical to this process to build multi-racial partnerships of people with a common under- 
standing of the problem and who are committed to antiracist community organizing. Mobilizing relational and expert power and eventually gaining the support of the formal power structure is critical to the systems change process. The final phase of reflection allows people engaged in shifting power in organizations or communities to consider the effectiveness of the strategies they have employed. It also includes considering lessons learned, reflecting on how power was wielded, and considering future directions for the organizing efforts.

In addition to the organizational level changes, dismantling racism is designed to increase individual knowledge, analytical skills, emotional faculties, and capacity to address institutional racism, social injustices and racial inequities within the organization and community (Watts, Williams, \& Jagers, 2003). This process is called sociopolitical development. These individual-level competencies can be thought of as processes and outcomes (Watts, Griffith, \& Abdul-Adil, 1999). As a process, individual-level change is characterized by three phases that represent important milestones for participants in anti-racist community organizing efforts: "(1) development of a more potent sense of self in relation to the world; (2) construction of more critical comprehension of the social and political forces which comprise one's daily life and world; and (3) cultivation of functional strategies and resources for attainment of personal or collective socio-political roles", (Serrano-García, 1984, p. 175). Part of this process is helping people to examine their political socialization, or how their individual thoughts, needs, and values have been formed in the context of a given sociopolitical system (Flanagan \& Gallay, 1995; MartínBaró, 1994).

As an outcome, sociopolitical development represents an individual-level change that is characterized by people developing a better understanding of themselves, and power in organizational and institutional settings. To create organizational or institutional change, it is critical that people understand two key sources of power - status and social power (Lamertz \& Aquino, 2004). Status is a person's relative standing or position based primarily on their title or position, and social power is a person's perceived ability to influence someone else's beliefs, attitudes and behavior as a function of the social resources he or she commands (Lamertz \& Aquino, 2004). Understanding these sources of power helps people consider who needs to be part of key discussions and decisions for creating organizational and institutional change.

In addition to understanding sources of power, it is important that people understand two types of power formal and informal. Formal power is associated with the position one holds in a formal organizational structure, including one's reporting relationships and the ability to issue (or be the catalyst for issuing) rewards and punishments (Lamertz \& Aquino, 2004; Morgan, 1997). Informal power is based on valued attributes and social resources people are perceived to have, including knowledge, skills, and interpersonal relationships (Lamertz \& Aquino, 2004). Because formal and informal power are often concentrated within a few people in a system, understanding the values and behavior of the people who can most effectively get things done and who are gatekeepers for others are critical to explicating the problems and solutions in a given system.

In addition to understanding where power resides and how it is derived, it is important for people to learn how power is exercised. Power is exercised through overt decision-making, agenda setting and prioritization, and shaping meaning, ideology and worldview (Freire, 1970; Gaventa, 1980; Gaventa \& Cornwall, 2001; Grassroots Policy Project, 2004; Terry, 1975). It is important that people understand how and what decisions are made, the power inherent in deciding what should be on an agenda for discussion, and how the issues on the agenda are prioritized. It also is critical for people to learn how issues are shaped and framed for discussion (e.g., see Daniels and Schulz (2006) for a discussion of the framing of health disparities), and the professional and personal ideologies and worldviews that serve as the context for decisions. Fundamental to dismantling racism is helping individuals who are part of an organization to recognize how organizations utilize power to create, perpetuate, and maintain power inequities.

\section{Soft Systems Methodology}

Soft Systems Methodology (SSM) is a methodological and theoretical approach that is most useful when addressing complex social problems yet methodological rigor and deep insights are needed (Midgley, 2006; Williams, 2005). Soft Systems Methodology emphasizes how moral decisions and ethical dilemmas are fundamental to understanding complex problems and designing and implementing interventions to change systems. The goal of SSM is to focus more critical thought about the world as it is versus how it might be, examining the situation in such a way that new learning emerges. Through SSM, participants are able to consider aspects of the world that were previously unexamined.

The SSM approach provides a critical rationale and process for deciding how and where to intervene in an organization to address healthcare disparities. Because it is highly unlikely that all stakeholders will agree on a conceptualization of the root cause of healthcare disparities or a level or strategy of intervention, SSM structures critical reflection and facilitates the development of strategies to 
promote systems change. Within the SSM approach Critical Systems Thinking has emerged to be a useful strategy to create systems change when there are multiple assumptions and logics about the root causes of the issue. This version of SSM is particularly useful in designing and implementing organizational and systems change when there are a number of stakeholders with different goals and whose values, assumptions, and perspectives need to be disentangled (Williams, 2005). The goal of designing and implementing a strategy to change the system can be achieved by acknowledging and developing different perspectives about the problem and the intervention, and then constructing models that articulate these perspectives and that compare the viewpoints with real life. These perspectives build towards a common understanding of the problem and the identification of possible relevant systems or places to intervene within the system to achieve the common goals. Critical Systems Thinking has been particularly effective for engaging people with no experience of planning and professionals, assuming that the plans, goals, and process are discussed in plain English (Midgley, 2006).

The dismantling racism intervention described below was developed through a process of adapting and integrating a conceptualization of institutional racism, antiracist community organizing, and Soft Systems Methodology. Based on our conceptualization of the problem, the goal of reducing racial healthcare disparities, and the healthcare organizational context, we have drawn the following five conclusions that drive the intervention approach described next: (1) modern healthcare disparities are rooted in a history of systems of racism and inequality that cut across institutions and levels within institutions; (2) power inequities are a fundamental aspect of racism in organizations, and creating accountability and more equitable distribution of power is a key to reducing healthcare disparities; (3) the cognitive and skill development of individuals is necessary but insufficient for addressing institutional racism; (4) institutional change requires a coordinated, multi-level intervention that leads to measurable change; and (5) strategies to address problems involving communities of color should incorporate an understanding of racism.

\section{Elements of a dismantling racism approach}

Dismantling racism is a systems change intervention designed to change the underlying infrastructure within an institution to be more fair, just and equitable. The overall goal of the dismantling racism intervention is to create an organization in which it can be demonstrated and it is perceived that all who seek health services are given equal, high quality care. The dismantling racism process has four objectives: (1) increase the accountability of individuals and systems to create a system for monitoring the elimination of healthcare disparities; (2) reorganize power by strengthening interpersonal relationships within the organization; (3) develop a common language and analytic framework for understanding the problem; and (4) create opportunities for individual growth and professional development. We will discuss each objective in turn.

Increase infrastructure, accountability, and monitoring

The first step in the dismantling racism process is the creation of a Change Team. The Change Team is the racially and professionally diverse team of leaders that guide the development, implementation and evaluation of the processes and outcomes of the dismantling racism intervention. The Change Team is a multi-racial group that represents organizational administration and staff, dismantling racism consultants, evaluators, community residents, and other relevant organizational system members. The Change Team leads the organization toward actively supporting (or at least avoid resisting) the changes necessary to move the organization toward its vision of becoming an anti-racist organization that provides the same high quality health services to all of its clients. The Change Team helps the organization integrate its mission of providing high quality health services with becoming an antiracist organization through the critical examination of its policies and procedures.

The infrastructure of the Change Team and the work it does is essential to making systems change. The Change Team guides the process of changing organizational culture and policies that can have an effect on the staff, the organization, and the extraorganizational system and environment. Their efforts are focused on making sure the overall organizational system and culture shifts, not just that individuals or problematic policies change.

The Change Team also is charged with monitoring and addressing policies and practices, resource allocations, relational structures, organizational norms and values, and individual skills and attitudes, as well as the root cause of racial healthcare disparities - racism. Thus, a major role they play is coordinating the collection, analysis and dissemination of data. These data are critical to the process as they provide strategies for understanding where and how to intervene at each level. It is critical to get data on job satisfaction, perceived racial climate, perceived cultural competence of staff, adequacy of resources for staff, job stressors, client and staff demographics, adequacy of policies and procedures, and organizational needs and challenges. This is not an exhaustive list, but simply 
illustrates the breadth of data that are useful. Where possible and feasible, these data should be collected from staff, clients and other stakeholders. Much of the data that is useful for monitoring healthcare disparities is already collected by the organization, or could be extracted from existing data (e.g., evaluating patient satisfaction based on patient demographics, and demographic discordance and concordance).

Develop a common language and analytic framework

Because racism is a complex, embedded, divisive, and often misunderstood construct, it is essential that there is a common definition and understanding of racism and how it affects healthcare quality. Thus, one of the core elements of the dismantling racism process is a "Dismantling Racism" or "Undoing Racism" workshop conducted by an anti-racist training organization (e.g., Dismantling Racism Works or The People's Institute for Survival and Beyond). The workshop is designed to provide a common language and conceptualization of racism. The goal is not indoctrination into a specific ideology, but to provide a vocabulary to facilitate communication and understanding. The workshop is designed for all who are part of a given system: community members, administrative staff members, service providers, board members, etc. Once people complete the workshop, they are encouraged, but not required, to participate in caucuses and, later, the Change Team.

Using adult learning principles and a popular education approach, this three-day workshop presents a sociohistorical analysis of the construction of race and racism in the US. In the first two days, the workshop includes a discussion and critical examination of how racism was constructed in the US, and how racism and discrimination are systematically carried out through the major institutions in society. The workshop also explores how racism is internalized as privilege and supremacy for Whites or is internalized as inferiority for People of Color. Central to this model is an analysis of where power resides in institutions and communities. The training highlights the role of institutional gatekeepers or those who determine access to organizations, institutions, and resources (Shapiro, 2002). It focuses on building accountable leadership and gatekeepers, and helping participants develop a critical view of the more prevalent, less visible systemic dimensions of racism. This individual-level intervention is a necessary foundation for organizational and larger systems-level interventions as it gives everyone a common language and understanding of root definitions and history on which to build.

A third workshop day is devoted to Anti-Racist Organizational Development. This innovation developed by
Dismantling Racism Works ${ }^{1}$ highlights the importance of using anti-racist values and principles to examine how the organization's culture, norms, policies and procedures can perpetuate or help to eliminate racism. A goal of this aspect of the training is helping workshop participants to recognize the possible incongruence between well-intentioned organizational processes, practices, and goals, and healthcare disparities. The organizational development component helps organizational stakeholders consider the complexity of systems problems, and highlights the need to focus change at the organizational level, not staff knowledge, attitudes, and behaviors. Finally, this aspect of the training introduces the five goals of anti-racist organizational development: (a) helping people who are committed to equity and anti-racist organizational values establish norms and a culture where people hold each other accountable for their behavior and the impact of their actions; (b) creating a culture where decisions about the allocation and use of money and resources consider their implications for social equity; (c) fostering organizational norms where decisions about how and what work gets done consider the racial equity; and (d) prioritizing of organizational goals and objectives is congruent with anti-racist organizational values. By introducing these key elements of organizational development and the notion of systems change, the dismantling racism workshop promotes an intervention strategy where the focus and goals remain on systems change.

\section{Reorganize power by strengthening relationships}

Dismantling Racism Works also developed a strategy to provide participants with ongoing opportunities after the workshop to struggle with and reflect on what they learned and channel it into individual and organizational level change. This primarily takes the form of "caucusing." A caucus is a gathering of people from a specific identity group who come together to support each other and address issues that are unique to that identity group. In the dismantling racism process, White people and People of Color participate in separate caucuses, which then are brought together to discuss common issues. People are organized in this manner based on the premise that racism affects Whites and People of Color in the US in very different ways. The caucuses provide healing and support for dealing with difficult and unique issues of identity and internalized superiority or oppression. Caucuses also provide

\footnotetext{
${ }^{1}$ Dismantling Racism Works is a group of community organizers who have pioneered adapting anti-racist organizing principles and strategies to organizations and institutions. Dismantling Racism Works also examines if and how the organization supports the empowerment of People of Color and anti-racist White allies.
} 
opportunities to plan, discuss, debate, and solve problems across racial lines.

In addition to these group activities, the dismantling racism intervention provides several opportunities for building relationships. One-on-one meetings and interviews are conducted with key members of the organization and community as part of an assessment and relationship building strategy. The Change Team creates a list of all key organizational stakeholders, then each Change Team member has a one-on-one meeting with each person. These meetings help to achieve two primary aims: (1) they provide a way to establish or build on individual relationships that may help to increase participation in the dismantling racism process; and (2) they provide a way to assess perceptions of the intervention from those who are part of the intervention, but also from those who are not. Creating opportunities for those who are not actively part of the dismantling racism process to give feedback provides critical data on barriers to systems change. Dispelling myths and misinformation about the process or addressing individual barriers can provide an important way of increasing participation and support for the dismantling racism process. These meetings also are coupled with " "member sharing." Member sharing is a structured opportunity for Change Team and caucus participants to get to know one another more personally by giving them the opportunity to share something personal. These opportunities are important strategies to humanize people, allowing those who may have initially viewed themselves as different to see how they are in fact similar. This provides an important and strong foundation of trust, which is important for creating space for people to examine their individual values, attitudes and beliefs around race, racism, and their specific job or profession.

The norms that are established by the training and the trust that is built among caucus participants are important cornerstones to the dismantling racism intervention. For staff members to grapple with how racism affects their organization and their professional practice, it is important to first establish a common understanding of the problem that is rooted in the different experiences of the participants. One of the fundamental elements of a systems change intervention is the examination of multiple perspectives, and the need to consider the unique lenses people bring to the organization. It is important for staff and clients to understand each other's perspective, and for White staff and staff of color to explore their common and unique experiences as well. It is through this understanding that people are able to more fully assess their blind spots and appreciate the complexity of healthcare disparities. Though much is gained from understanding these individual perspectives, it is equally important for staff, clients, and other key stakeholders to understand how intraorga- nizational and extraorganizational factors influence the quality of healthcare.

Create opportunities for individual growth and professional development

In addition to the organizational-level goals, the dismantling racism process is committed to creating individuallevel change as well. The efforts to educate staff, make systems and decisions more transparent, diversify leadership opportunities, and enhance accountability are all goals affecting individuals as well as the organization. The individual-level goals are to increase people's awareness of how their personal experiences, histories, beliefs and values may influence their work, specifically the provision of healthcare or other services within a healthcare organization. An additional goal is to increase people's willingness to take risks and leadership within the organization and the community. The Change Team and caucuses are designed to foster and develop new organizational leaders by providing opportunities and supports for those lower on the professional hierarchy to have the opportunity to be leaders. Increasing the diversity of informal leadership is critical to the dismantling racism process, particularly given the challenges and limitations of focusing on staff diversity. If the other elements of the dismantling racism intervention are put in place, a nurturing environment for personal growth and organizational change will be cultivated.

\section{Discussion}

This article proposes a conceptual and methodological approach for creating healthcare systems change and eliminating healthcare disparities called dismantling racism. Healthcare disparities have persisted across disease, time, and type of care. These racial differences are rooted in a long and complex history of racism in society, medicine and healthcare. Though most directly measured by assessing racial differences in the quality of services provided by providers, healthcare disparities are not simply the result of healthcare providers' individual misbehavior or miseducation. The extent and persistence of healthcare disparities suggest that racial differences in healthcare quality are rooted in institutional inequities that are entrenched in the healthcare system. Thus, addressing healthcare disparities requires a systemic approach that can capture the breadth and complexity of relevant issues, yet organize a realistic strategy for addressing the problem.

Dismantling racism provides a strategy for moving from the organizational and institutional factors that are at the root of healthcare disparities to an intervention that creates and sustains organizational and institutional service equity. 
The conceptual and theoretical foundation of the approach integrates institutional racism, anti-racist community organizing, and Soft Systems Methodology. No one of these theories is sufficiently comprehensive to describe all necessary aspects of the problem, context and intervention. The strength of the dismantling racism intervention is that it provides a strategy for stakeholders of diverse racial and ethnic backgrounds, professional positions, and other relationships with the organization to collaborate on the strategy to eliminate healthcare disparities. It begins with recognition and respect for the diverse perspectives that people will have on the root of the problem and the intervention strategy. The goal of the systems change approach is to build a common vision of what the organization might be in comparison to what it currently is. By structuring this critical examination, new learning occurs and all stakeholders are able to consider aspects of the problem, organization, and society that were previously unexamined (Midgley, 2006; Williams, 2005).

The dismantling racism intervention approach begins with creating a racially and professionally diverse Change Team. This group is the foundation of the intervention, and they guide the implementation of the intervention. They are also the body that holds the rest of the organization accountable to the goal of providing all clients with equally good, high quality care by collecting, monitoring, and feeding back a diverse array of data that are proximal and distal to healthcare disparities. Central to this process is developing a common language and analytic framework through the workshop. This training provides a critical basis for understanding the systems framework of both the problem and the intervention. In addition to the Change Team infrastructure and the common understanding fostered by the training, interpersonal relationships are potentially the most important key to a successful intervention. It is critical that the Change Team creates a safe setting for honest dialogue and disclosure of individual and collective struggles to grow personally and professionally. Individual growth and professional development occur, at least sometimes, within interpersonal settings, particularly as the collective struggles help to humanize people and build strong trusting relationships. These relationships are what holds the organization together and helps create and sustain change. These diverse components are combined to create and sustain change in the organizational system, not just to change one face of the problem.

Dismantling racism is unique in its direct focus on oppression at multiple levels within an organizational system. Eliminating, or at least reducing, healthcare disparities requires developing strategies that are congruent with each level of racism and that consider different faces, types, and levers of power. At the extraorganizational level, this could include helping staff within an organization to build relationships with people in other organizations, in and outside of healthcare, and to strengthen the organization's relationship with its constituencies (Speer and Hugley, 1995). These relationships utilize social power and informal relationships to exercise various forms of power. These relationships can be particularly helpful in framing the discussion and setting the agenda around these issues in the community, professional circles, and in the organization. By strategically illustrating to diverse organizational stakeholders how addressing racism or creating equitable systems of care is congruent with the organization's mission and goals, it is possible to build support to address these issues among those who might otherwise not be interested in such an initiative. It is imperative that these bodies of stakeholders are given an opportunity to share an understanding of the problem and data to help them monitor organizational change. Particularly to sustain the intervention, it is essential that the organizational leadership is able to demonstrate the existence of the problem and how the intervention is making progress toward the goal, even if the progress is in the form of impact rather than outcome evaluation data.

At the intraorganizational level, it is essential to demonstrate how organizational policies and procedures, even when not intended, may contribute to healthcare disparities. Developing a common conceptual understanding of the problem and data that is congruent with a socially and contextually accurate understanding of the problem is also important. Staff and clients need to understand how racism functions in a healthcare system, and ideally how it is functioning in their organization. Monitoring and evaluation data are essential to helping divisions of the organization and the organization's leadership track the problem and their success towards its eradication. It is important to simultaneously promote standards of high quality care, consistent with the most recent service guidelines, and address the areas where there are gaps in the quality of care provided.

Finally, at the individual level, it is important to provide data and interventions that will promote and reinforce services that are of the highest quality to all. Individuallevel interventions should only begin after interventions at the intraorganizational and extraorganizational level have been conducted. Because individual behavior is shaped and promoted by organizational culture and practice, it is essential to first uncover the intraorganizational factors that may contribute to or reinforce healthcare disparities before focusing on individual-level change. Once this foundation is articulated, it is important to be able to collect data on the provision of care by providers, and aggregate the data according to patient demographic characteristics. Being able to provide these data promotes accountability and allows for more focused intervention. Individual level 
interventions to address healthcare disparities must address not only technical skills, but the social attitudes, values and beliefs that consciously and unconsciously affect healthcare services. Raising awareness of differences, providing data on the uniqueness of cultural groups, and even evidence-based guidelines are not likely to eliminate healthcare disparities, though they may decrease them. Because healthcare disparities are rooted in racism, it is important to address how racism has influenced and is a part of individuals in order to effectively change how individuals provide services.

Despite the strengths of this intervention approach, there are some noteworthy limitations. In general, when compared with educational or traditional organizational interventions, the comprehensive nature of Dismantling Racism makes it more labor intensive, complex and difficult. Systems change interventions, like dismantling racism, are also designed to address large, socially charged, and seemingly intractable problems, which often lack unanimous support. Thus, spending the time up front to understand the problem within a given organizational and systemic context is invaluable. Though it is not practical or recommended that this become a lengthy process, it is essential to invest the time to understand the different aspects of institutional racism, perceptions of healthcare disparities from multiple perspectives, and come to agreement on short and long-term goals. Systems-level interventions, particularly those to address racism or other forms of oppression, should be developed thoughtfully, deliberately and carefully. Addressing issues that are so engrained in our culture and institutions, not to mention individual values and beliefs, is, to understate it, difficult. It is important to anticipate passive and active resistance and hostility. This opposition from stakeholders may adversely affect individuals' job satisfaction, job stability, interpersonal relationships, and the organizational climate.

Before beginning a dismantling racism intervention, it is critical to assess the organizational environment first. In a multi-racial or multi-ethnic organizational environment, it is critical to begin by identifying People of Color and Whites who may be willing to commit to the process. If the organizational or community context is racially or ethnically diverse, it is important to assess different levels of interest and investment, perspectives on the perceived feasibility and timing of the intervention, and perceived risk of such an approach. For example, what are the outcomes of interest to the organization's leadership, funders, and other stakeholders? Is the political and social climate one where racism could be discussed openly? What other forms of oppression are important to consider in this context? How can people who are willing to participate be protected from misuse of power, inaccurate accusations of racism or actual racism? Since it is reasonable to assume all will not be on board with any process that includes discussion of racism or oppression, what type of backlash could you anticipate and from whom? Each of these issues is critical to consider before engaging in such a change process.

Also, in interventions to address racism and other forms of oppression, there is often a tendency to view the education and training as an end, not a process. This is a mistake. One of the most important features of systems change interventions, like dismantling racism, is the vigilant focus on the system. Though individual change, discovery and competence are important, it is critical to help people to remain focused on systems change, and to continue to view individual-level factors within the organizational and systemic context. Though potentially counterintuitive, because racism and oppression are so difficult and emotionally charged, it can be immobilizing or engender feelings of hopelessness and powerlessness when there are not positive goals as the markers of success. For example, if individuals acceptance of labels (i.e., White people admitting they are 'racist' because they benefit from institutional racism) becomes the marker of change and success, the struggle with the cognitive dissonance of accepting such a negative label can derail the process and divert attention from the larger goal of organizational and systems change. Racism is only useful as a frame for systems change if it remains focused on characteristics of the system. The leadership of the dismantling racism intervention must be vigilant about focusing on organizational policies, procedures, and practices, not individual flaws or scapegoats.

To address institutional racism, it is important to begin addressing the complex and multiple causes and faces of oppression, and the many ways it is affecting individuals, organizations, institutions, and communities. Having people with different perspectives on the problem collaborate on addressing it is critical for accurate problem identification and creating a solid foundation for systems change. The causes of problems, particularly pervasive problems like healthcare disparities, are often larger than individuals. Systemic problems are rooted in institutions, making institutional oppression an important concept to consider when addressing any form of organizational, institutional, or community change. Creating systems change to address racism and other forms of institutional oppression is not a linear process, nor can all of the necessary change strategies be anticipated from the beginning. Nevertheless, systems change interventions that are guided by the values and principles of social equity can help interventions and organizations eliminate racism and other forms of oppression, even when it is not always obvious what the intervention strategies should be. As Dr. King notes: “...the line of progress is never straight. For a period a movement may 
follow a straight line and then it encounters obstacles and the path bends. It is like curving around a mountain when you are approaching a city. Often it feels as though you were moving backward, and you lost sight of your goal; but in fact you are moving ahead, and soon you will see the city again, closer by" (King, 1967 as cited in Washington, 1986, p. 562).

Acknowledgements This research was supported in part by the W.K. Kellogg Foundation Community Health Scholars Program (now called the W.K. Kellogg Health Disparities Program) and by federal grants: 1-U48-DP-000055, R06/CCR421449-01, and R25GM05864106 . Its contents are solely the responsibility of the authors and do not necessarily represent the official views of the Centers for Disease Control and Prevention or the National Institutes of Health. We gratefully acknowledge Julie Allen for her editorial assistance and the work of Dismantling Racism Works (formerly Changework), the Rural County Public Health Department Dismantling Racism Change Team, the Cancer Care and Racial Equity Study team, and The Partnership Project.

\section{References}

Agency for Healthcare Research and Quality. (2005). National healthcare disparities report. Rockville, MD: Agency for Healthcare Research and Quality.

Aptheker, H. (1992). Anti-Racism in U.S. History: The first two hundred years. Westport, CT: Greenwood Press.

Baquet, C. R., Carter-Pokras, O., \& Bengen-Seltzer, B. (2004). Healthcare disparities and models for change. American Journal of Managed Care, 10 Spec No, SP5-SP11.

Blanchard, J., \& Lurie, N. (2004). R-E-S-P-E-C-T: patient reports of disrespect in the health care setting and its impact on care. Journal of Family Practice, 53(9), 721-730.

Byrd, W. M., \& Clayton, L. A. (2000). An american health dilemma. New York: Routledge.

Byrd, W. M., \& Clayton, L. A. (2001). Race, medicine, and health care in the United States: A historical survey. Journal of the National Medical Association, 93(Suppl. 3), 11S-34S.

Ceci, S. J., \& Papierno, P. B. (2005). The rhetoric and reality of gap closing: when the "havenots" gain but the "haves" gain even more. American Psychologist, 60(2), 149-160.

Daniels, J., \& Schulz, A. J. (2006). Constructing whiteness in health disparities research. In A. J. Schulz (Ed.), Gender, race, class, and health: Intersectional approaches. (pp. 89-127). San Francisco, CA: Jossey-Bass.

Escarce, J. J., \& McGuire, T. G. (2004). Changes in racial differences in use of medical procedures and diagnostic tests among elderly persons: 1986-1997. American Journal of Public Health, 94(10), $1795-1799$.

Freeman, H. P. (2003). Commentary on the meaning of race in science and society. Cancer Epidemiology, Biomarkers and Prevention, 12(3), 232s-236s.

Flanagan, C., \& Gallay, L. S. (1995). Reframing the meaning of "political" in research with adolescents. Perspectives on Political Science, 24(1), 34-42.

Freire, P. (1970). Pedagogy of the oppressed. New York: Herder and Herder.

Gaventa, J. (1980). Power and powerlessness: Quiescence and rebellion in an Appalachian valley. Oxford: Clarendon Press.

Gaventa, J., \& Cornwall, A. (2001). Power and knowledge. In P. Reason \& H. Bradbury (Eds.), Handbook of action research:
Participative inquiry and practice. (pp. 70-80). Thousand Oaks, CA: Sage Publications.

Geiger, H. J. (2006). Health disparities: What do we know? What do we need to know? What should we do? In A. Schulz \& L. Mullings (Eds.), Gender, race, class and health: Intersectional approaches (pp. 261-288). San Francisco, CA: Jossey Bass.

Gillborn, D. (2005). Education policy as an act of white supremacy: Whiteness, critical race theory and education reform. Journal of Education Policy, 20(4), 485-505.

Grassroots Policy Project. (2004). Power and organizing. Retrieved 3 August 2004, from http://www.grassrootspolicy.org/publications/power.html.

Green, L. A., Gorenflo, D. W., \& Wyszewianski, L. (2002). Validating an instrument for selecting interventions to change physician practice patterns: a Michigan Consortium for Family Practice Research study. Journal of Family Practice, 51(11), 938-942.

Griffith, D.M., Childs, E.L., Eng, E., \& Jeffries, V. (2007). Racism in organizations: The case of a county public health department. Journal of Community Psychology, 35(3), 291-306.

Griffith, D. M., Moy, E., Reischl, T. M., \& Dayton, E. (2006). National data for monitoring and evaluating racial and ethnic health inequities: Where do we go from here? Health Education and Behavior, 33(4), 470-487.

Hahn, M. P., Ostermann, P. A., Richter, D., \& David, A. (1995). [Classification, therapy and complications of pediatric femoral neck fractures]. Zentralblatt für Chirurgie, 120(11), 832-840.

Horner, R. D., Salazar, W., Geiger, H. J., Bullock, K., Corbie-Smith, G., \& Cornog, M. et al. (2004). Changing healthcare professionals' behaviors to eliminate disparities in healthcare: What do we know? How might we proceed? American Journal of Managed Care, 10 Spec No, SP12-SP19.

Johnson, R. L., Saha, S., Arbelaez, J. J., Beach, M. C., \& Cooper, L. A. (2004). Racial and ethnic differences in patient perceptions of bias and cultural competence in health care. Journal of General Internal Medicine, 19(2), 101-110.

Jones, J. M. (1997). Prejudice and racism (2nd ed.). New York: McGraw-Hill Companies.

Jones, K. J. (2003). Community organizing. A workbook for organizers working to build a movement for social change. Jacksonville, F.L.: Change Work Organizational Development Consultants.

King, G. (1996). Institutional racism and the medical/health complex: a conceptual analysis. Ethnicity and Disease, 6(1-2), 30-46.

King, M. L. (1966). National convention of the medical committee for human rights. Chicago, IL, (March 25).

Kressin, N. R., \& Petersen, L. A. (2001). Racial differences in the use of invasive cardiovascular procedures: review of the literature and prescription for future research. Annals of Internal Medicine, 135(5), 352-366.

Krieger, N. (1987). Shades of difference: theoretical underpinnings of the medical controversy on black/white differences in the United States, 1830-1870. International Journal of Health Services, 17(2), 259-278.

Lamertz, K., \& Aquino, K. (2004). Social power, social status and perceptual similarity of workplace victimization: A social network analysis of stratification. Human Relations, 57(7), $795-822$.

LaVeist, T. A., Nickerson, K. J., \& Bowie, J. V. (2000). Attitudes about racism, medical mistrust, and satisfaction with care among African American and white cardiac patients. Medical Care Research Review, 57(Suppl. 1), 146-161.

Lavizzo-Mourey, R., \& Jung, M. (2005). Fighting unequal treatment: the Robert Wood Johnson Foundation and a quality-improvement approach to disparities. Circulation, 111(10), 1208-1209.

Lillie-Blanton, M., Maddox, T. M., Rushing, O., \& Mensah, G. A. (2004). Disparities in cardiac care: rising to the challenge of 
Healthy People 2010. Journal of the American College of Cardiologists, 44(3), 503-508.

MacPherson, W. (1999). The stephen lawrence inquiry. London: Stationery Office Limited.

Martín-Baró, I., Aron, A., \& Corne, S. (1994). Writings for a liberation psychology. Cambridge, MA: Harvard University Press.

Midgley, G. (2006). Systemic intervention for public health. American Journal of Public Health, 96(3), 466-472.

Morgan, G. (1997). Images of organization (2nd ed.). Thousand Oaks, Calif.: Sage Publications.

Neighborhood Funders Group. (2001). Community organizing: The basics. <http://www.nfg.org/cotb/07whatisco.htm.>. Accessed, November 1. Neighborhood Funders Group.

Pettigrew, T. F. (2004). Justice deferred a half century after Brown v. Board of education. American Psychologist, 59(6), 521-529.

Saha, S., Arbelaez, J. J., \& Cooper, L. A. (2003). Patient-physician relationships and racial disparities in the quality of health care. American Journal of Public Health, 93(10), 1713-1719.

Serrano-Garcia, I. (1984). The illusion of empowerment: Community development within a colonial context. Prevention in Human Services, 3, 173-200.

Shavers, V. L., \& Brown, M. L. (2002). Racial and ethnic disparities in the receipt of cancer treatment. Journal of the National Cancer Institute, 94(5), 334-357.

Shapiro, I. (2002). Training for racial equity and inclusion: A guide to selected programs. Washington, DC: The Aspen Institute.

Smedley, B. D., Stith, A. Y., \& Nelson, A. R. (2003). Unequal treatment: Confronting racial and ethnic disparities in health care. Washington, D.C.: National Academies Press.

Smith, D. B. (1999). Health care divided: Race and healing a nation. Ann Arbor: University of Michigan Press.

Sohler, N., Walmsley, P. J., Lubetkin, E., \& Geiger, H. J. (2003). Equal Treatment: an annotated bibliography of studies on race and ethnic disparities in healthcare, their cause, and related issues. New York, NY: Physicians for Human Rights.

Speer P. W., \& Hughey J. (1995). Community organizing: An ecological route to empowerment and power. American Journal of Community Psychology, 23(5), 729-748.
Stevens, G. D., \& Shi, L. (2002). Racial and ethnic disparities in the quality of primary care for children. Journal of Family Practice, $51(6), 573$.

Sullivan Commission on Diversity in the Healthcare Workforce. (2004). Missing persons: Minorities in the health professions. A report of the Sullivan Commission on Diversity in the Healthcare Workforce. Atlanta, GA: The Sullivan Commission.

Taylor, S. L., \& Lurie, N. (2004). The role of culturally competent communication in reducing ethnic and racial healthcare disparities. American Journal of Managed Care, 10 Spec No, SP1SP4.

Terry, R. W. (1975). For Whites Only (Rev. ed.). Grand Rapids: Eerdmans. Dismantling Institutional Racism 36.

Trivedi, A. N., Zaslavsky, A. M., Schneider, E. C., Ayanian, J. Z. (2005). Trends in the quality of care and racial disparities in medicare managed care. New England Journal of Medicine, 353(7), 692-700.

Trubek, L. G., \& Das, M. (2003). Achieving equality: healthcare governance in transition. American Journal of Law and Medicine, 29(2-3), 395-421.

Watts, R. J., Griffith, D. M., \& Abdul-Adil, J. (1999). Sociopolitical development as an antidote for oppression. American Journal of Community Psychology, 27(2), 255-271.

Watts, R. J., Williams, N. C., \& Jagers, R. J. (2003). Sociopolitical development. American Journal of Community Psychology, 31(1-2), 185-194.

Williams, B. (2005). Soft systems methodology. Retrieved 25 February 2006 at http://users.actrix.co.nz/bobwill.

Williams, D. R., \& Rucker, T. D. (2000). Understanding and addressing racial disparities in health care. Health Care Financing Review, 21(4), 75-90.

Wittig, M.A. (1996). An introduction to social psychological perspectives on grassroots organizing. Journal of Social Issues, $52(1), 3-14$.

Wyszewianski, L., \& Green, L. A. (2000). Strategies for changing clinicians' practice patterns. A new perspective. Journal of Family Practice, 49(5), 461-464. 